\title{
Deep learning based computer-aided diagnosis for neuroimaging data: focused review and future potential
}

\author{
V. B. Surya Prasath ${ }^{1,2,3}$ \\ ${ }^{1}$ Computational Imaging and VisAnalysis (CIVA) Lab, Department of Computer Science, University of Missouri-Columbia, \\ Columbia, MO 65211, USA. \\ ${ }^{2}$ Division of Biomedical Informatics, Cincinnati Children's Hospital Medical Center (CCHMC), Cincinnati, OH 45229, USA. \\ ${ }^{3}$ Department of Biomedical Informatics, College of Medicine, University of Cincinnati, OH 45267, USA.
}

Correspondence to: Dr. V. B. Surya Prasath, Computational Imaging and VisAnalysis (CIVA) Lab, Department of Computer Science, University of Missouri-Columbia, Columbia, MO 65211, USA.E-mail: prasaths@missouri.edu

How to cite this article: Prasath VBS. Deep learning based computer-aided diagnosis for neuroimaging data: focused review and future potential. Neuroimmunol Neuroinflammation 2018;5:1. http://dx.doi.org/10.20517/2347-8659.2017.68

Received: 25 Dec 2017 Accepted: 28 Dec 2017 Published: 12 Jan 2018

Science Editor: Athanassios P. Kyritsis Copy Editor: Lu Liu Production Editor: Huan-Liang Wu

Automatic image analysis techniques applied to neuroimaging data in general, and magnetic resonance imaging (MRI), and functional MRI (fMRI) in particular, have proven to be effective computer-aided diagnosis (CAD) tools in neuroscience ${ }^{[1-4]}$. Recently, the advancements in machine learning techniques combined with the wide availability of computational power have proven to be efficient in solving previously difficult problems in analyzing neuroimaging data. At the forefront of these advancements is the usage of deep (artificial) neural network architectures that led robust learning based techniques to attack challenging problems such as segmentation and classification in neuroimaging data $^{[5-8]}$.

Many of the impressive results obtained in CAD using deep learning (DL) techniques utilize mainly image datasets. DL networks typically require annotations of several images for employing supervised learning techniques and are one of the roadblocks in employing these state of the art networks in various classification tasks in MRI/fMRI. However, unsupervised learning techniques within the DL paradigm are now being employed in natural image classification with a lot of success and we believe the adaptability of these to the neuroimaging data are required to attack challenging neuroimage analysis problems.

A stacked denoising auto encoders approach that is an unsupervised learning technique was used ${ }^{[9]}$ for brain tumor segmentation in MRI imagery. The experimental results showed that using this particular approach is as good as using supervised learning based DL techniques that require accurate image-based annotations. This indicates that we can use different unsupervised learning in DL networks variants for

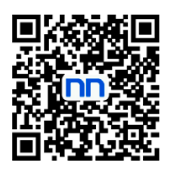


various neuroimaging data problems. A Siamese DL networks approach ${ }^{[10]}$ for detecting spinal metastasis with a multi-resolution technique correctly detected $100 \%$ of lesions on a dataset of 26 sagittal MR images from 14 males and 12 females $(58 \pm 14$ years; mean \pm SD). The DL network considered produced only 0.40 false positives (FPs) per case. Further, at a true positive (TP) rate of 90\%, with aggregation FPs were reduced from $0.375 \mathrm{FPs}$ per case to $0.207 \mathrm{FPs}$ per case obtaining $44.8 \%$ overall reduction. Although this work was for MR images of the spine, the usage of a Siamese neural network with the aggregation strategy promises to be an interesting approach that can also be adapted to brain MRI/fMRI imagery.

Utilizing domain-transfer convolutional neural networks, an end-to-end DL technique ${ }^{[1]}$, shows great promise since it overcomes the following problems of traditional classification and other DL based methods: (1) the need for manual design of feature space; (2) effective feature vector classifier or segment specific detection object and image patches; (3) large training datasets; (4) computing resources; and (5) long waiting times for training a perfect deep model. An example classification of the Open Access Series of Imaging Studies (OASIS)-MRI dataset showed good potential for such an approach's generalizability.

Extreme learning machines is a variant of DL networks, and an application in resting state fMRI data for schizophrenia was undertaken ${ }^{[12]}$ and experimental results indicated that near $90 \%$ accuracy was obtained on a dataset of 72 patient images and 75 healthy controls (18 to 65 years) from the Center for Biomedical Research Excellence (COBRE)'s raw anatomical and fMRI data on this difficult classification problem. A DL pipeline ${ }^{[13]}$ applied to recognize Alzheimer's disease using fMRI data obtained overall highest accuracy of $96.86 \%$ on 28 patient images and 15 healthy controls ( 24 female and 19 male, $74.9 \pm 5.7$ years) from the Alzheimer's Disease Neuroimaging Initiative (ADNI) dataset.

Most of the CAD pipelines with DL techniques at their core utilize non-medical data to train due to the lack of availability of massive labeled data. Recent advancements in natural image analysis with DL methods are yet to be used for neuroimaging data and the challenges in obtaining the datasets/ annotations/labels, improvising/adapting DL networks, parameters setup, multi-modality generalization pose remain to be solved. However, the recent advancements in deep learning based image analysis shows great potential for analyzing MRI/fMRI imagery. Even with the limited results available so far in the literature, with deep learning based CAD for neuroimaging data we believe the future is bright for solving some of the hard neuroimage analysis problems.

\section{DECLARATIONS}

\section{Authors' contributions}

Prasath VBS contributed solely to this letter.

Financial support and sponsorship

None.

\section{Conflicts of interest}

There are no conflicts of interest.

\section{Patient consent}

Not applicable.

\section{Ethics approval}

Not applicable.

\section{Copyright}

(c) The Author(s) 2018. 


\section{REFERENCES}

1. Pelapur R, Prasath VBS, Moreno JC, Heck MM. 3D Workflow for segmentation and interactive visualization in brain MR images using multiphase active contours. Proceedings of the IEEE International Conference on Bioinformatics and Biomedicine (BIBM); Kansas, USA; 2017. p. 911-6

2. Kalavathi P, Senthamilselvi M, Prasath VBS. Review of computational methods on brain symmetric and asymmetric analysis from neuroimaging techniques. Technologies 2017;5:16.

3. Kalavathi P, Prasath VBS. Automatic segmentation of cerebral hemispheres in MR human head scans. Int J Imaging Syst Technol 2016;26:15-23.

4. Kalavathi P, Prasath VBS. Adaptive nonlocal filtering for brain MRI restoration. In: Thampi S, Bandyopadhyay S, Krishnan S, Li KC, Mosin S, Ma M, editors. Advances in signal processing and intelligent recognition systems. Advances in intelligent systems and computing. Cham: Springer; 2015. p. 571-80.

5. Greenspan H, van Ginneken B, Summers RM. Deep learning in medical imaging: overview and future promise of an exciting new technique. IEEE Trans Medical Imag 2016;35:1153-9.

6. Yonekura A, Kawanaka H, Prasath, VBS, Aronow BJ, Takase H. Glioblastoma multiforme tissue histopathology images based disease stage classification with deep CNN. Proceedings of the 6th International Conference on Informatics, Electronics \& Vision (ICIEV); 2017.

7. Yonekura A, Kawanaka H, Prasath, VBS, Aronow BJ, Takase, H. Improving the generalization of disease stage classification with deep CNN for glioma histopathological images. Proceedings of the 2017 IEEE International Conference on Bioinformatics and Biomedicine (BIBM); Kansas, USA; 2017. p. 1222-6.

8. Yonekura A, Kawanaka H, Prasath, VBS, Aronow BJ, Takase H. Disease stage classification for Glioblastoma Multiforme histopathological images using deep convolutional neural network. Proceedings of Joint 17th World Congress of International Fuzzy Systems Association and 9th International Conference on Soft Computing and Intelligent Systems (IFSA-SCIS); Otsu, Japan; 2017.

9. Vaidhya K. Unsupervised and semi-supervised deep learning for medical imaging. Anthill Inside. Madras, India: Predible Health; 2017.

10. Wang J, Fang Z, Lang N, Yuan H, Su MY, Baldi P. A multi-resolution approach for spinal metastasis detection using deep Siamese neural networks. Comp Bio Med 2017;84:137-46.

11. Pang S, Yu Z, Orgun MA. A novel end-to-end classifier using domain transferred deep convolutional neural networks for biomedical images. Comp Meth Prog Biomed 2017;140:283-93.

12. Chyzhyk D, Savio A, Graña M. Computer aided diagnosis of schizophrenia on resting state fMRI data by ensembles of ELM. Neural Networks 2015;68:23-33.

13. Sarraf S, Tofighi G. Deep learning-based pipeline to recognize Alzheimer's disease using fMRI data. Proceedings of Future Technologies Conference (FTC); 2016. p. 816-20. 